\title{
CCY-1a-E2 induces G2/M phase arrest and apoptotic cell death in HL-60 leukemia cells through cyclin-dependent kinase 1 signaling and the mitochondria-dependent caspase pathway
}

\author{
CHIN-FEN LIN ${ }^{1 *}$, JAI-SING YANG $^{2 *}$, CHINGJU LIN ${ }^{3}$, FUU-JEN TSAI $^{4,5}$, \\ CHI-CHENG LU ${ }^{6}$ and MIAU-RONG LEE ${ }^{1}$
}

\begin{abstract}
${ }^{1}$ Department of Biochemistry, China Medical University, Taichung 404; ${ }^{2}$ Department of Medical Research, China Medical University Hospital, China Medical University, Taichung 404; ${ }^{3}$ Department of Physiology, China Medical University, Taichung 404; ${ }^{4}$ Human Genetic Center, China Medical University Hospital, Taichung 404; ${ }^{5}$ School of Post-Baccalaureate Chinese Medicine, China Medical University, Taichung 404;

${ }^{6}$ School of Nutrition and Health Sciences, Taipei Medical University, Taipei 110, Taiwan, R.O.C.
\end{abstract}

Received March 7, 2016; Accepted April 22, 2016

DOI: $10.3892 /$ or.2016.4970

\begin{abstract}
Our previous study demonstrated that 2-[(3-methoxybenzyl)oxy]benzaldehyde (CCY-1a-E2) is a potent compound that acts against multiple human leukemia cell lines. CCY-1a-E2 was also shown to have efficacious anti-leukemic activity in vivo. However, the molecular mechanism of action of CCY-1a-E2 attributed to its anticancer effect remains poorly understood. In the present study, CCY-1a-E2 suppressed cell viability in multiple leukemia cell lines (HL-60, K562, KG-1 and KG-1a) via inhibition of cell proliferation, cell cycle arrest and induction of apoptosis. CCY-1a-E2 exhibited a marked toxic effect on HL-60 cells and displayed low cytotoxicity in normal human peripheral blood mononuclear cells (PBMCs). Results from flow cytometric analysis indicated that CCY-1a-E2 promoted G2/M phase arrest and promoted apoptosis in the HL-60 cells. CCY-1a-E2 treatment upregulated cyclin $\mathrm{B}$, cyclin-dependent kinase 1 (CDK1), cell division cycle $25 \mathrm{C}$ (cdc25C) and p21 protein expression. CCY-1a-E2 caused apoptotic cell death and DNA fragmentation as determined by 4',6-diamidino-2-phenylindole (DAPI) staining and DNA gel electrophoresis. Elevated activities of caspase-8, -9 and -3 were observed during CCY-1a-E2-induced cell apoptosis; their specific inhibitors were found to block CCY-1a-E2-induced apoptosis, respectively. Moreover, CCY-1a-E2 time-dependently disrupted the mitochondrial membrane potential $(\Delta \Psi \mathrm{m})$, and it enhanced the protein levels
\end{abstract}

Correspondence to: $\mathrm{Dr}$ Miau-Rong Lee, Department of Biochemistry, China Medical University, 91 Hsueh-Shih Road, Taichung 404, Taiwan, R.O.C.

E-mail:mrlee@mail.cmu.edu.tw

*Contributed equally

Key words: 2-benzyloxybenzaldehyde, CCY-1a-E2, G2/M phase arrest, apoptosis, leukemia cells of Fas/CD95, cytochrome $c$, Bax, cleaved PARP, as well as attenuated $\mathrm{Bcl}-2$ expression in the HL-60 cells. Our results provide direct evidence that supports the future potential therapeutic application of CCY-1a-E2 in leukemia.

\section{Introduction}

Leukemia is characterized by uncontrolled cell proliferation and blockage in the differentiation of hematopoietic cells $(1,2)$. However, clinical trials concerning treatment strategies for leukemia have not achieved satisfactory outcomes, and new targets for treating leukemia are necessary. One of the best strategies for new anti-leukemia agents are carried out via induction of cell differentiation or apoptotic death in leukemia cells (3-5). Regulation and/or management of cell cycle progression and apoptosis are prominent approaches to anti-leukemia therapy $(2,6)$. Cyclin-dependent kinase (CDK) complexes can modulate cell cycle progression, especially cyclin-dependent kinase 1 (CDK1) and cyclin $\mathrm{B}$ are pivotal molecules in the regulation of the cell cycle in the $\mathrm{G} 2 / \mathrm{M}$ phase $(7,8)$. The cell division cycle $25 \mathrm{C}(\mathrm{cdc} 25 \mathrm{C})$ phosphatase controls CDK1 activity and accelerates mitosis entry by dephosphorylation of CDK1 on Thr14 and Tyr15 sites $(9,10)$. Additionally, the activity of CDK1/cyclin B complex is blocked by $\mathrm{p} 21^{\mathrm{wa} / \mathrm{cip} 1}$ signaling which serves as a CDK inhibitor $(11,12)$. Several agents have been shown to interfere with the activity of CDK1 and cause subsequent cell cycle arrest, and these agents have been developed into significant clinical anticancer drugs through induction of cancer cell apoptosis $(13,14)$. When tumor cells undergo apoptosis, nuclear condensation, DNA fragmentation and apoptotic bodies are manifested $(15,16)$. During the apoptotic process, caspase proteins undergo proteolytic processing and trigger a cascade of caspase activation $(17,18)$. Therefore, these key factors can regulate the apoptotic process and play a vital role in the treatment of leukemia.

Benzyloxybenzaldehyde derivatives have been reported to have multiple biological functions, including binding to estrogen receptors (ER $\alpha$ and $\beta$ ), arresting cell cycle 
progression and inducing apoptotic cell death (19-21). A class of 2-benzyloxybenzaldehyde derivatives was designed and synthesized in our laboratory (20). 2-Benzyloxybenzaldehyde analog 2-[(3-methoxybenzyl)oxy]benzaldehyde (CCY-1a-E2) has been found to be a potent compound against leukemia cells. CCY-1a-E2 exhibited an anti-leukemic effect on a leukemia BALB/c mouse model (19). High dosage treatment $(100 \mathrm{mg} / \mathrm{kg})$ of CCY-1a-E2 was found to have no adverse effects on renal, hepatic and hematological parameters upon safety evaluation analysis (19). However, the molecular mechanism underlying the anti-leukemia effects of CCY-1a-E2 has not been completely clarified. Here, the anti-proliferative activity of CCY-1a-E2 was evaluated in human leukemia HL-60 cells. We found that CCY-1a-E2 led to cell cycle arrest at the G2/M phase and caused apoptosis in HL-60 cells through mitochondria-dependent caspase cascade signaling.

\section{Materials and methods}

Chemicals and reagents. Fetal bovine serum (FBS), L-glutamine, penicillin-streptomycin, RPMI-1640 medium, and trypsin-EDTA were obtained from Thermo Fisher Scientific, Inc. (Waltham, MA, USA). The primary antibodies used in this study and their corresponding $\operatorname{IgG}$ antibodies conjugated to horseradish peroxidase (HRP) were purchased from Santa Cruz Biotechnology, Inc. (Santa Cruz, CA, USA). Z-IETD-FMK (a specific caspase-8 inhibitor), Z-LEHD-FMK (a specific caspase-9 inhibitor) and Z-DEVD-FMK (a specific caspase-3 inhibitor) were obtained from R\&D Systems, Inc. (Minneapolis, MN, USA). All chemicals and reagents were of analytical grade and purchased from Sigma-Aldrich Corp. (St. Louis, MO, USA) unless otherwise stated.

Cell culture. Human promyelocytic leukemia cell line HL-60 and human acute myelogenous leukemia cell lines KG-1 and $\mathrm{KG}-1 \mathrm{a}$ were purchased from the Bioresource Collection and Research Center (BCRC) (Hsinchu, Taiwan). K562 erythroleukemia cell line was purchased from the American Type Culture Collection (ATCC) (Manassas, VA, USA). Peripheral blood mononuclear cells (PBMCs) were collected from whole blood samples with the BD Vacutainer Mononuclear Cell Preparation Tube (CPT) with sodium heparin (Becton, Dickinson and Co., Franklin Lakes, NJ, USA) and were isolated using Ficoll-Paque ${ }^{\mathrm{TM}}$ Plus (GE Healthcare UK, Ltd., Little Chalfont, Buckinghamshire, UK). Cells were placed into $75-\mathrm{cm}^{2}$ culture flasks and were grown in RPMI-1640 medium supplemented with $10 \%$ FBS, $100 \mathrm{U} / \mathrm{ml}$ penicillin, and $100 \mu \mathrm{g} / \mathrm{ml}$ streptomycin at $37^{\circ} \mathrm{C}$ under a humidified atmosphere of $5 \% \mathrm{CO}_{2}$ and $95 \%$ air.

Detection of cell number and viability. HL-60, K562, KG-1 and KG-1a cells $\left(1 \times 10^{4}\right.$ cells/well) in 96-well plates were incubated with $0,1,2.5,5$ and $10 \mu \mathrm{M}$ of CCY-1a-E2 for 24 and $48 \mathrm{~h}$. The trypan blue dye exclusion assay was applied to determine the number of viable cells by using a Countess Automated Cell Counter (Thermo Fisher Scientific, Inc.) as previously reported (22). HL-60 cells were exposed to $0,1,2.5,5$ and $10 \mu \mathrm{M}$ of CCY-1a-E2 after pre-incubation with or without 10,25 and $50 \mu \mathrm{M}$ of Z-IETD-FMK, Z-LEHD-FMK and Z-DEVD-FMK for
2 h. 3-(4,5-Dimethylthiazol-2-yl)-2,5-diphenyltetrazolium bromide (MTT) assay was performed for the quantitative analysis of cell viability as described elsewhere $(23,24)$.

Assessment of cell cycle distribution by flow cytometric analysis. HL-60 cells $\left(2 \times 10^{5}\right.$ cells/well) were seeded into 12-well plates and then treated with $5 \mu \mathrm{M}$ CCY-1a-E2 for $0,3,6,12$ and $24 \mathrm{~h}$. The cells were fixed and stained with propidium iodide (PI) solution following a previously reported method (6). The sub-G1 peak (apoptotic population) and cell cycle profiling were determined using a BD FACSCalibur flow cytometer (BD Biosciences, San Jose, CA, USA), and the data were analyzed by utilizing BD CellQuest software.

Immunoblotting analysis. HL-60 cells $\left(1 \times 10^{7}\right.$ cells) were placed in $\mathrm{T} 75$ flasks and treated with $5 \mu \mathrm{M}$ CCY-1a-E2 for 0 , $1,2,4,6,8,10,12$ and $16 \mathrm{~h}$. After treatments, the cells were lysed with lysis buffer, and each sample was electrophoresed as previously detailed $(15,22,25)$, and the membrane was probed with an appropriate secondary antibody for enhanced chemiluminescence (Immobilon Western HRP Substrate; Merck Millipore, Bedford, MA, USA).

4',6-Diamidino-2-phenylindole (DAPI) staining and DNA fragmentation assay. HL-60 cells $\left(2 \times 10^{5}\right.$ cells $\left./ \mathrm{ml}\right)$ were treated with $5 \mu \mathrm{M}$ CCY-1a-E2 for $24 \mathrm{~h}$ and thereafter stained with $1 \mu \mathrm{g} / \mathrm{ml}$ DAPI as previously described (16,25). After a 48-h exposure, DNA was extracted from each sample and electrophoresis was run according to a previous method (26).

Analyses of caspase-3, -8 and -9 activities. HL-60 cells at a density of $1 \times 10^{7}$ cells/flask were incubated with $5 \mu \mathrm{M}$ CCY-1a-E2 for 0, 2, 4, 8 and $12 \mathrm{~h}$. At the end of the incubation, the cells were lysed and assessed according to the manufacturer's instructions provided in the Caspase-3, -8 and -9 Colorimetric Assay kits (R\&D Systems, Inc.).

Determination of mitochondrial membrane potential ( $\Delta \Psi m)$. HL-60 cells at a density of $2 \times 10^{5}$ cells/well in 12 -well plates were treated with $5 \mu \mathrm{M}$ CCY-1a-E2 for $0,6,12,18$ and $24 \mathrm{~h}$. Cells from each treatment were harvested and re-suspended in $500 \mu \mathrm{l}$ of $\mathrm{DiOC}_{6}$ (3) (Thermo Fisher Scientific, Inc.) at $50 \mathrm{nM}$ for $\Delta \Psi \mathrm{m}$. After incubation at $37^{\circ} \mathrm{C}$ for $30 \mathrm{~min}$, the cells were analyzed by flow cytometry as described by Lee et al (27).

Statistical analysis. The statistical significance of the difference was defined $(\mathrm{p}<0.05)$ and carried out utilizing Student's $\mathrm{t}$-test, and the data are expressed as the mean \pm standard deviation (SD) from three independent experiments.

\section{Results}

CCY-1a-E2 reduces cell viability in leukemia cells. Four cell lines (HL-60, K562, KG-1 and KG-1a) were used to assess the cytotoxicity of CCY-1a-E2. The cells were treated with $0,1,2.5$, 5 and $10 \mu \mathrm{M}$ of CCY-1a-E2 for $24 \mathrm{~h}$, and the viable cell number was measured by trypan blue dye exclusion assay. CCY-1a-E2 dose-dependently decreased the viability of the HL-60, K562, KG-1 and KG-1a cells. Notaby, HL-60 cells were more sensitive to CCY-1a-E2 than the three other cell lines (Fig. 1A). 
A

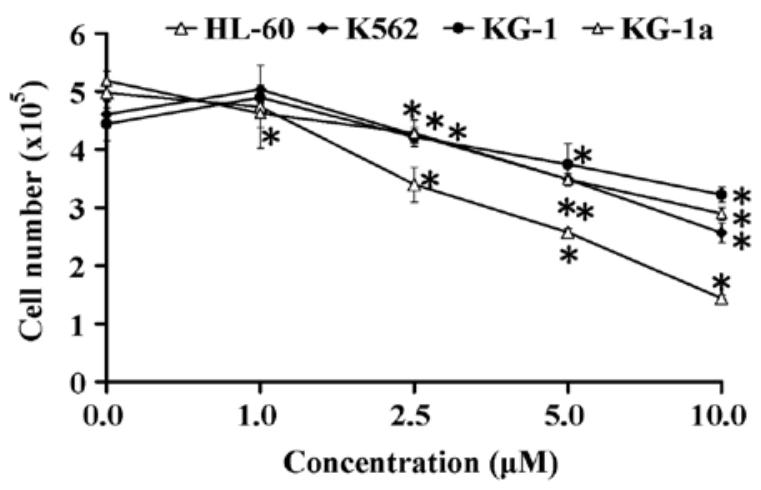

B

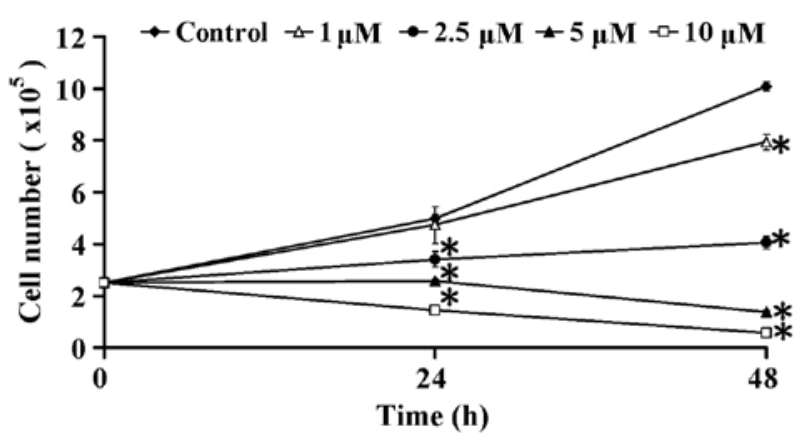

Figure 1. CCY-1a-E2 inhibits cell number in multiple leukemia cell lines. (A) HL-60, K562, KG-1 and KG-1a cells were incubated with various concentrations of CCY-1a-E2 for $24 \mathrm{~h}$. (B) Time-dependent effects of CCY-1a-E2 on HL-60 cells. Cells were measured as described in 'Materials and methods'. Each point corresponds to the mean $\pm \mathrm{SD}(\mathrm{n}=3),{ }^{*} \mathrm{p}<0.05$ vs. untreated control. SD, standard deviation.

In addition, the inhibitory effect of CCY-1a-E2 on the proliferation of HL-60 cells was time-dependent (Fig. 1B), and the half maximal inhibitory concentration $\left(\mathrm{IC}_{50}\right)$ value for the 48-h treatment of CCY-1a-E2 in the HL-60 cell line was $5.32 \pm 0.25 \mu \mathrm{M}$. In contrast, CCY-1a-E2 exerted low cytotoxicity against normal human PBMCs (Fig. 2). Our results suggest that CCY-1a-E2 exhibits anti-leukemia action against HL-60 cells in vitro.

CCY-1a-E2 causes G2/M phase arrest in HL-60 cells. To explore the effect of CCY-1a-E2 on cell cycle distribution, the cells were treated for various time periods with CCY-1a-E2. The percentage of treated cells in phase G1, S and G2/M was detected by DNA content stained with PI. CCY-1a-E2 induced $\mathrm{G} 2 / \mathrm{M}$ phase arrest and increased the sub-G1 population (apoptotic cells) (Fig. 3A). Exposure of HL-60 cells to $5 \mu \mathrm{M}$ CCY-1a-E2 for 6, 12 and $24 \mathrm{~h}$ resulted in a significant increase in the percentage of cells in the G2/M phase, while a marked decrease in the percentage of cells in the G0/G1 phase was observed (Fig. 3B). To examine the protein levels associated with the G2/M phase, CCY-1a-E2-treated cells were analyzed by immunoblotting. The protein expression levels of cyclin B, CDK1, cdc25C were increased. However, after 6 hours of treatment, cdc25C protein level gradually decreased, but p21 protein level significantly increased (Fig. 4). These find-

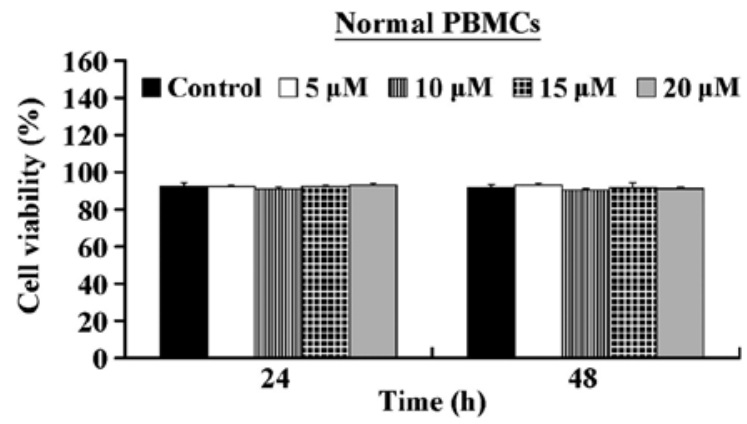

Figure 2. CCY-1a-E2 has no effect on the viability of normal PBMCs. Normal PBMCs were incubated with various concentrations of CCY-1a-E2 for 24 and $48 \mathrm{~h}$, and the cell viability was measured by MTT assay as described in 'Materials and methods'. The results are presented as the means \pm SD $(n=3)$. PBMCs, peripheral blood mononuclear cells; SD, standard deviation.
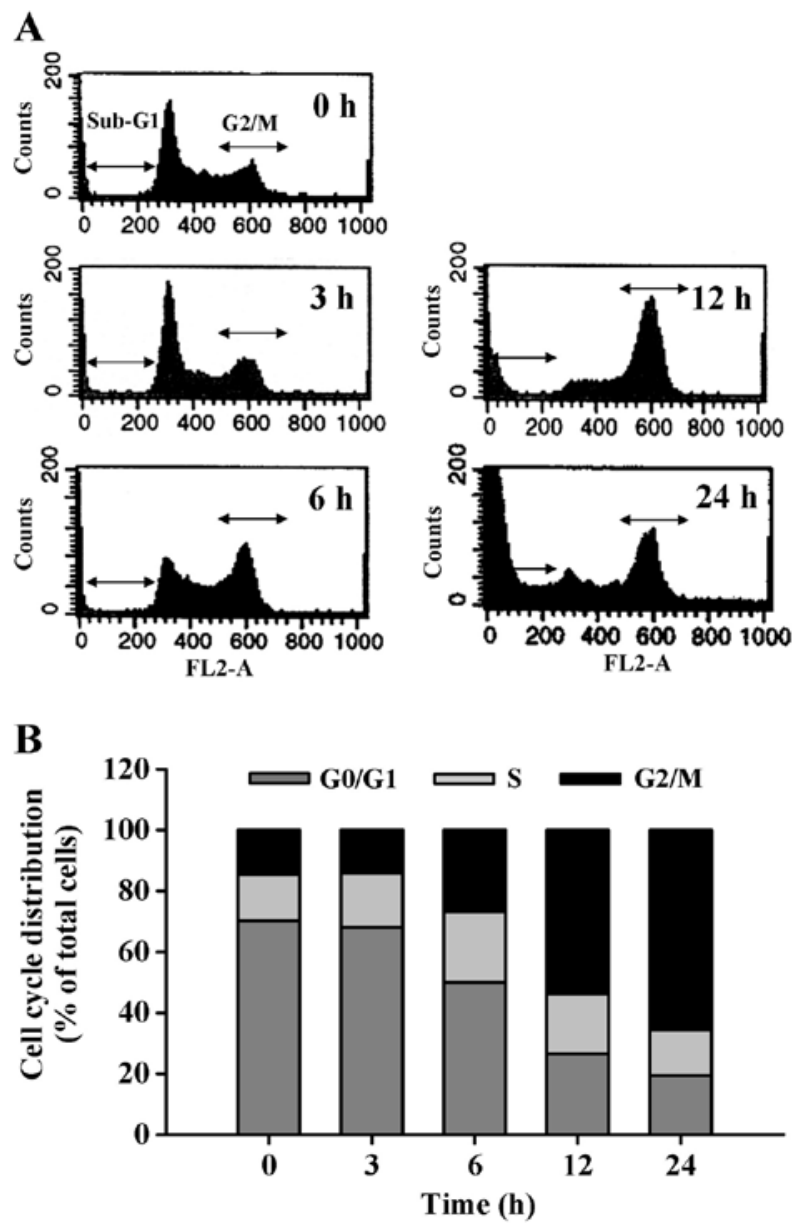

Figure 3. CCY-1a-E2 enhances G2/M phase arrest and apoptosis in HL-60 cells. Cells were incubated for $5 \mu \mathrm{M}$ CCY-1a-E2 for the indicated time intervals. (A) Cell cycle distribution was determined by PI staining and immediately analyzed by flow cytometry. Results are shown of representatives of three independent experiments. (B) Cell cycle profile of the CCY-1a-E2-treated cells was quantified, and the percentages of cells in the G0/G1, S and G2/M phases are shown. PI, propidium iodide.

ings suggest that CCY-1a-E2 regulated CDK1 activation and resulted in G2/M phase arrest in the HL-60 cells.

CCY-1a-E2 triggers apoptotic death in HL-60 cells. In Fig. 3, the percentage of cells in the sub-G1 phase (apoptotic cells) 


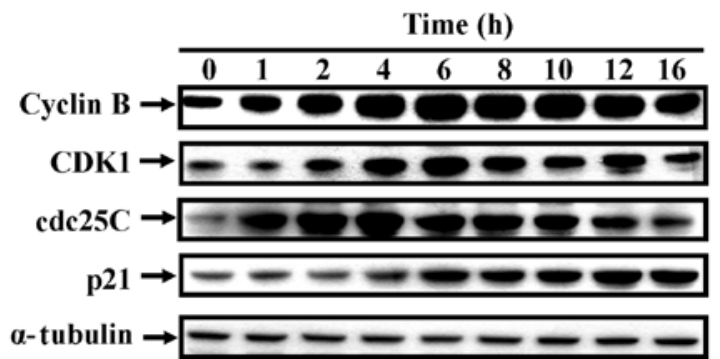

Figure 4. CCY-1a-E2 alters the expression of G2/M phase-associated proteins in the HL-60 cells. The cells were treated with $5 \mu \mathrm{M}$ CCY-1a-E2 for the indicated time intervals. The protein levels of cyclin $\mathrm{B}, \mathrm{CDK} 1, \mathrm{cdc} 25 \mathrm{C}$ and p21 were examined by western blot analysis. $\alpha$-tubulin was used as the internal standard for all immunoblots. CDK1, cyclin-dependent kinase 1; cdc25C, cell division cycle 25C.

A
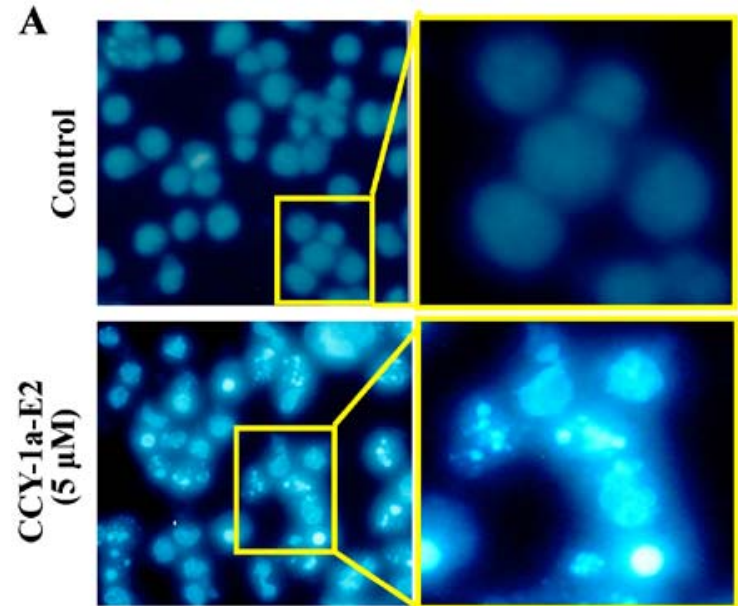

B

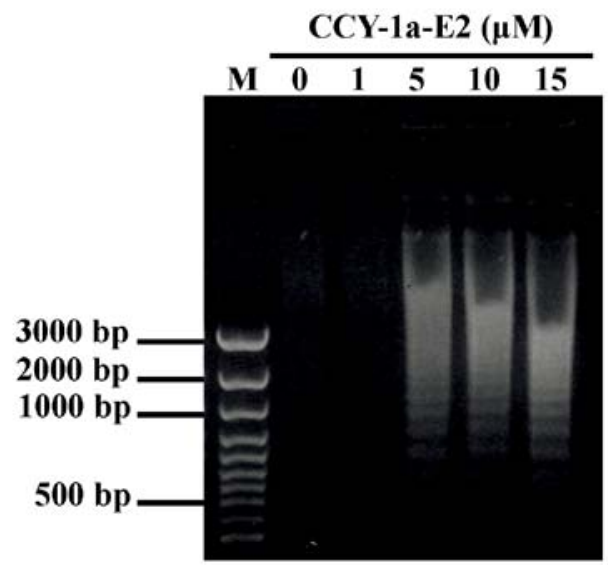

Figure 5. CCY-1a-E2 triggers apoptosis in the HL-60 cells. The cells were incubated with different concentrations of CCY-1a-E2 for $24 \mathrm{~h}$. (A) The cells were then stained with DAPI and examined under a fluorescence microscope. (B) DNA extracts were electrophoresed in $1.0 \%$ agarose gel, and subsequently stained with $\mathrm{EtBr}$ for $30 \mathrm{~min}$ as described in 'Materials and methods'. EtBr, ethidium bromide.

was increased after CCY-1a-E2 exposure. To evaluate whether CCY-1a-E2 induces apoptosis, we performed DAPI staining for chromatin condensation and a DNA fragmentation assay. The number of cells with brighter cell nuclei were elevated in the CCY-1a-E2-treated cells (Fig. 5A). In addition, DNA was extracted from the cells following treatment with various
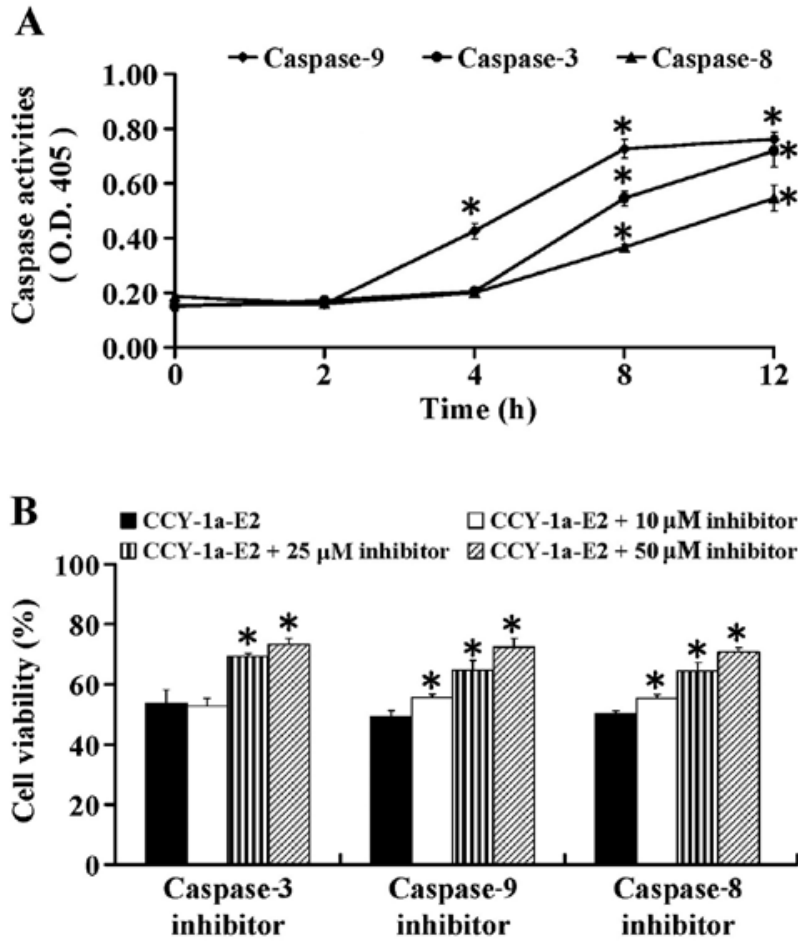

Figure 6. CCY-1a-E2 stimulates the activation of caspase-9, -8 and -3 in the HL-60 cells. Cells were incubated with $5 \mu \mathrm{M}$ CCY-1a-E2 for the indicated periods of time. (A) The activities of caspase-9, -3 and -8 were measured as described in 'Materials and methods'. (B) Cells were pre-treated with different concentrations of caspase-3 inhibitor (Z-DEVD-FMK), caspase-9 inhibitor (Z-LEHD-FMK) and caspase-8 inhibitor (Z-IETD-FMK) for $2 \mathrm{~h}$ and then exposed to $5 \mu \mathrm{M}$ CCY-1a-E2 for $24 \mathrm{~h}$. Cell viability was determined by MTT assay. The results are expressed as the mean \pm SD $(n=3)$, *p $<0.05$ vs. CCY-1a-E2 treatment. SD, standard deviation.

concentrations of CCY-1a-E2 for $24 \mathrm{~h}$, and subjected to agarose gel electrophoresis. DNA ladder was observed in samples treated with 5, 10 and $15 \mu \mathrm{M}$ CCY-1a-E2 (Fig. 5B). Our results imply that CCY-1a-E2 treatment induced apoptosis in the HL-60 cells.

CCY-1a-E2 enhances caspase- $9,-8$ and -3 activities in HL-60 cells. To further ascertain whether CCY-1a-E2-induced apoptosis is caspase-dependent, we performed specific caspase activity assays. HL-60 cells were exposed to $5 \mu \mathrm{M}$ CCY-1a-E2 for different time periods. We found that CCY-1a-E2 enhanced the activities of caspase- $9,-3$ and -8 (Fig. 6A) after 2, 4, 8 and $12 \mathrm{~h}$ of exposure. To confirm the role of caspase-mediated apoptosis by CCY-1a-E2, the cells were pre-treated with specific caspase inhibitors. Our data showed that Z-IETD-FMK, Z-LEHD-FMK and Z-DEVE-FMK at 10,25 and $50 \mu \mathrm{M}$ concentration-dependently suppressed CCY-1a-E2-reduced cell viability (Fig. 6B). These data indicated that CCY-1a-E2-induced apoptosis was mediated via both extrinsic and intrinsic signaling in the HL-60 cells.

CCY-1a-E2 collapses $\triangle \Psi m$ and alters mitochondria-mediated apoptosis signaling in HL-60 cells. To confirm whether the CCY-1a-E2-provoked apoptosis was mediated via the mitochondrial pathway, the level of $\Delta \Psi \mathrm{m}$ was measured and immunoblotting was carried out. CCY-1a-E2 treatment led to a decrease in $\Delta \Psi \mathrm{m}$ in a time-course pattern (Fig. 7). CCY-1a-E2 


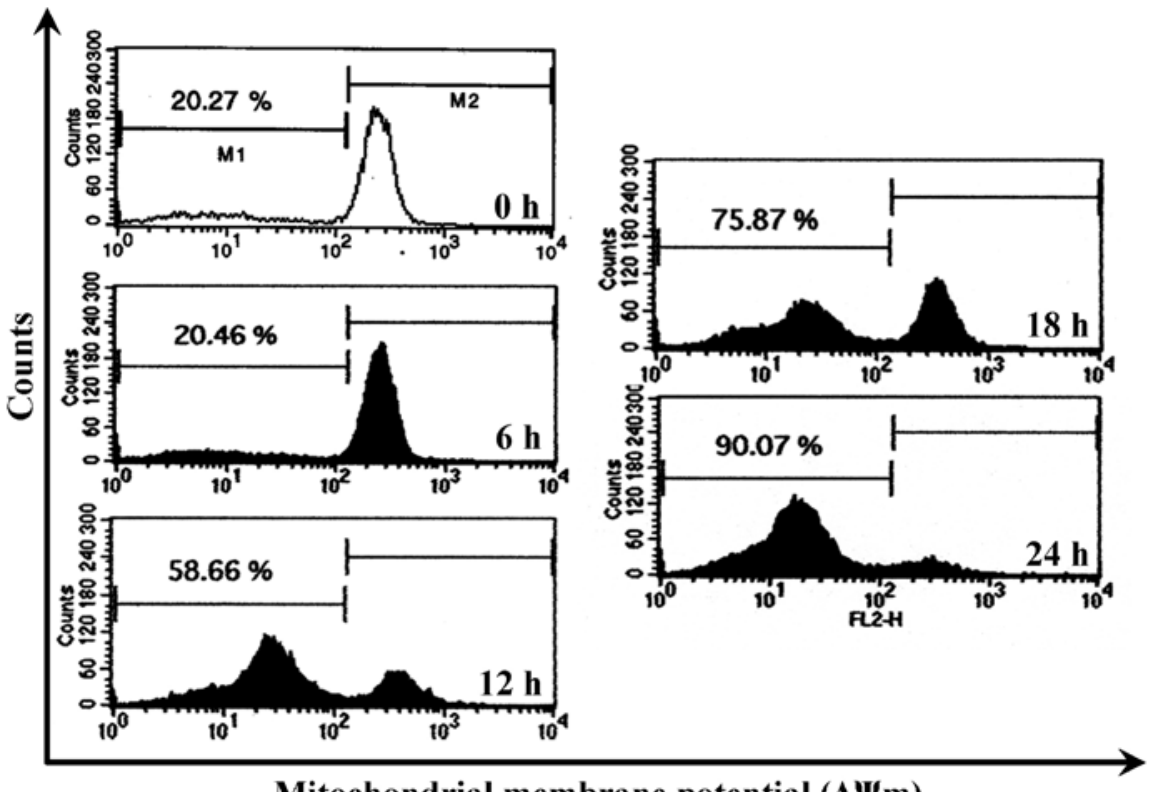

Figure 7. CCY-1a-E2 induces loss of $\Delta \Psi \mathrm{m}$ in the HL-60 cells. The cells were treated with $5 \mu \mathrm{M}$ CCY-1a-E2 for the indicated time periods. Cells were stained with the selective $\operatorname{DiOC}_{6}(3)$ dye to determine $\Delta \Psi \mathrm{m}$ by flow cytometry as described in 'Materials and methods'. $\Delta \Psi \mathrm{m}$, mitochondrial membrane potential

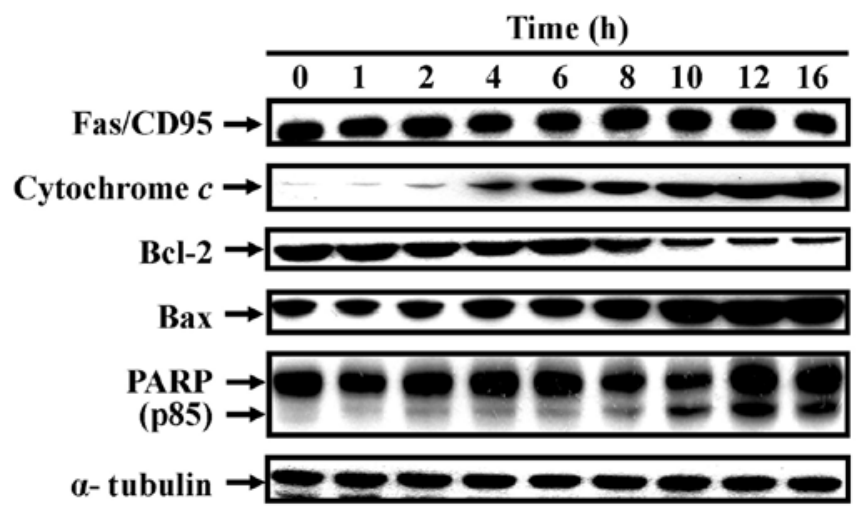

Figure 8. CCY-1a-E2 affects the levels of apoptosis-related proteins in the HL-60 cells. The cells were treated with $5 \mu \mathrm{M}$ CCY-1a-E2 for the indicated time intervals, and the total proteins were prepared and detected by western blot analysis as described in 'Materials and methods'. The blot was individually probed with the specific primary antibodies for Fas/CD95, cytochrome $c$, Bcl-2, Bax and PARP, and $\alpha$-tubulin served as an internal control.

also promoted the protein expression of cytochrome $c$, Bax and PARP, while it suppressed the level of Bcl-2 (Fig. 8). Additionally, activation of Fas/CD95 protein occurred in the treated cells (Fig. 8). Therefore, these results conclude that CCY-1a-E2-mediated apoptosis of HL-60 cells was carried out through the mitochondria- and death receptor-dependent pathway.

\section{Discussion}

2-Benzyloxybenzaldehyde (CCY-1a) has been shown to inhibit superoxide anion generation via Akt inactivation and phospholipase $\mathrm{D}$ activation in rat neutrophils (21). In addition, 2-benzyloxybenzaldehyde can block $\mathrm{Ca}^{2+}$ entry and suppress formyl peptide-stimulated increase in intracellular $\mathrm{Ca}^{2+}$ in neutrophils (28). Pan et al (29) demonstrated that 2-benzyloxybenzaldehyde modulates vascular smooth muscle cell proliferation by blocking the Ras/p42/44 MAPK pathway and inhibiting NF- $\mathrm{KB}$ and AP-1 DNA binding activities. Our previous study showed that CCY-1a-E2 reduced the percentage of viable murine leukemia WEHI-3 cells, and the $\mathrm{IC}_{50}$ value of CCY-1a-E2 was $5 \mu \mathrm{M}$ for a 24 -h treatment (19). To evaluate the anticancer effect of CCY-1a-E2 on other leukemia cell lines, we treated HL-60, K562, KG-1 and KG-1a cells with various concentrations of CCY-1a-E2. A significant concentration-dependent decrease of cell viability was observed in all cell lines after $24 \mathrm{~h}$ exposure to $2.5-10 \mu \mathrm{M}$ of CCY-1a-E2. CCY-1a-E2 not only decreased the cell viability in HL-60 cells, but also exerted low cytotoxicity in PBMCs (Figs. 1 and 2). The clinical applicability of an anti-leukemia drug depends on its being distinct in both its potency and therapeutic index between leukemia and normal blood cells (6). Our result was in agreement with the previous study of CCY-1a-E2 on reducing viability of WEHI-3 cells (19). Overall, CCY-1a-E2 represents a promising candidate as an anticancer agent for leukemia due to its low toxicity to normal cells.

CCY-1a-E2 significantly inhibited cell viability and induced cell apoptosis in the HL-60 cells (Figs. 1 and 3). However, the molecular mechanisms of its anti-leukemia activity remain unknown. Our data demonstrated that CCY-1a-E2 triggered cell cycle arrest at the G2/M phase after a 6-h exposure, while its effect on the sub-G1 cell population appeared following a 12-h treatment (Fig. 3A). This result indicated that CCY-1a-E2-induced G2/M phase arrest occurred before the onset of apoptosis. The CDK1/cyclin B complex is one of the main regulators, resulting in $\mathrm{G} 2 / \mathrm{M}$ progression and apoptosis. G2/M checkpoints can be exerted by inactivating cdc25C and activating CDK inhibitor (p21waf/cip), which subsequently inactivates CDK1, and prevents cells from entering mitosis $(9,12)$. In the present study, we investigated 
the expression levels of $\mathrm{G} 2 / \mathrm{M}$ phase-related proteins. Our results demonstrated that CCY-1a-E2 induced the expression of cyclin B, CDK1, cdc25C and p21 in a time-dependent manner (Fig. 4). Our finding implicates a novel role for the 2-benzyloxybenzaldehyde derivative.

Cells undergo apoptosis in response to cell death-inducing signals from death receptors on the cell surface [such as Fas, death receptor 4 (DR4) or DR5], mitochondria, or endoplasmic reticulum (ER) stress (30-32). During apoptosis, caspases are activated and arranged in a proteolytic cascade to convey the apoptotic signal $(17,18)$. Apoptotic evidence of chromatin condensation and DNA fragmentation was observed in the CCY-1a-E2-treated HL-60 cells, indicating that cells underwent apoptosis in response to CCY-1a-E2 treatment (Fig. 5). Moreover, induction of caspase-9, -8 and -3 activities (Fig. 6) as well as the specific cleavage of PARP (Fig. 8) were detected in the CCY-1a-E2-treated cells. Application of the intrinsic and extrinsic caspase specific inhibitors blocked the CCY-1a-E2-reduced cell viability (Fig. 6). This result further confirmed the effect of CCY-1a-E2 on apoptotic pathways. The caspase-independent factors such as apoptosis-inducing factor (AIF) and endonuclease G (Endo G) can be released from mitochondria into the cytosol $(33,34)$. Our results fail to exclude the possibility of a caspase-independent pathway involved in the CCY-1a-E2-induced apoptosis.

Loss of $\Delta \Psi \mathrm{m}$ is one of the features of apoptosis $(35,36)$. Indeed, we detected a significant loss of $\Delta \Psi \mathrm{m}$ in the CCY-1a-E2-treated HL-60 cells (Fig. 7). Moreover, cytochrome $c$ release is related to the change in $\mathrm{Bcl}-2$ family proteins during cell apoptosis. Bcl-2 and Bax are located in the mitochondrial outer membrane, and their ratio (Bcl-2/Bax) regulates the release of apoptotic elements (cytochrome $c$, pro-caspase-9, AIF and Endo G) to the cytosol $(18,31,35)$. Our data indicated that the protein expression levels of cytochrome $c$, Bax and PARP (p85) were increased, whereas the protein expression level of $\mathrm{Bcl}-2$ was decreased in the CCY-1a-E2-treated HL-60 cells (Fig. 8). Our results are consistent with these available observations as evidenced by the upregulation of Bax protein and the downregulation of Bcl-2 protein in the CCY-1a-E2-treated cells. These results suggest that the activation of the caspase cascade contributed to CCY-1a-E2-induced apoptosis of HL-60 cells.

In conclusion, we demonstrated that CCY-1a-E2 exerts cytotoxic activity against leukemia cells, while it is less toxic to normal PBMCs. CCY-1a-E2 induced G2/M phase arrest followed by caspase-mediated apoptosis in the human leukemia HL-60 cells. Taken together, these findings provide important new insights into the possible molecular mechanisms of the anti-leukemia activity of CCY-1a-E2.

\section{References}

1. Riether C, Schürch $\mathrm{CM}$ and Ochsenbein AF: Regulation of hematopoietic and leukemic stem cells by the immune system. Cell Death Differ 22: 187-198, 2015.

2. Lu CC, Yang JS, Chiang JH, Hour MJ, Lin KL, Lin JJ, Huang WW, Tsuzuki M, Lee TH and Chung JG: Novel quinazolinone MJ-29 triggers endoplasmic reticulum stress and intrinsic apoptosis in murine leukemia WEHI-3 cells and inhibits leukemic mice. PLoS One 7: e36831, 2012.

3. Wang W, Lv M, Zhao X and Zhang J: Developing a novel indolocarbazole as histone deacetylases inhibitor against leukemia cell lines. J Anal Methods Chem 2015: 675053, 2015.
4. Maioral MF, Gaspar PC, Rosa Souza GR, Mascarello A, Chiaradia LD, Licínio MA, Moraes AC, Yunes RA, Nunes RJ and Santos-Silva MC: Apoptotic events induced by synthetic naphthylchalcones in human acute leukemia cell lines. Biochimie 95: 866-874, 2013.

5. Padma VV: An overview of targeted cancer therapy. Biomedicine (Taipei) 5: 19, 2015.

6. Yang JS, Hour MJ, Huang WW, Lin KL, Kuo SC and Chung JG: MJ-29 inhibits tubulin polymerization, induces mitotic arrest, and triggers apoptosis via cyclin-dependent kinase 1-mediated Bcl-2 phosphorylation in human leukemia U937 cells. J Pharmacol Exp Ther 334: 477-488, 2010.

7. Kan SF, Huang WJ, Lin LC and Wang PS: Inhibitory effects of evodiamine on the growth of human prostate cancer cell line LNCaP. Int J Cancer 110: 641-651, 2004.

8. Murray AW: Recycling the cell cycle: Cyclins revisited. Cell 116: 221-234, 2004.

9. Timofeev O, Cizmecioglu O, Settele F, Kempf T and Hoffmann I: Cdc25 phosphatases are required for timely assembly of CDK1-cyclin B at the G2/M transition. J Biol Chem 285: 16978-16990, 2010.

10. Castedo M, Perfettini JL, Roumier T and Kroemer G: Cyclin-dependent kinase-1: Linking apoptosis to cell cycle and mitotic catastrophe. Cell Death Differ 9: 1287-1293, 2002.

11. Yang JS, Hour MJ, Kuo SC, Huang LJ and Lee MR: Selective induction of G2/M arrest and apoptosis in HL-60 by a potent anticancer agent, HMJ-38. Anticancer Res 24: 1769-1778, 2004.

12. Elsayed YA and Sausville EA: Selected novel anticancer treatments targeting cell signaling proteins. Oncologist 6: 517-537, 2001.

13. Shah MA and Schwartz GK: Cell cycle-mediated drug resistance: An emerging concept in cancer therapy. Clin Cancer Res 7: 2168-2181, 2001.

14. Chan KS, Koh CG and Li HY: Mitosis-targeted anti-cancer therapies: Where they stand. Cell Death Dis 3: e411, 2012.

15. Lu CC, Yang JS, Chiang JH, Hour MJ, Lin KL, Lee TH and Chung JG: Cell death caused by quinazolinone HMJ-38 challenge in oral carcinoma CAL 27 cells: Dissections of endoplasmic reticulum stress, mitochondrial dysfunction and tumor xenografts. Biochim Biophys Acta 1840: 2310-2320, 2014.

16. Chiang JH, Yang JS, Lu CC, Hour MJ, Chang SJ, Lee TH and Chung JG: Newly synthesized quinazolinone HMJ-38 suppresses angiogenetic responses and triggers human umbilical vein endothelial cell apoptosis through p53-modulated Fas/death receptor signaling. Toxicol Appl Pharmacol 269: 150-162, 2013.

17. Chung JG, Yang JS, Huang LJ, Lee FY, Teng CM, Tsai SC, Lin KL, Wang SF and Kuo SC: Proteomic approach to studying the cytotoxicity of YC-1 on U937 leukemia cells and antileukemia activity in orthotopic model of leukemia mice. Proteomics 7: 3305-3317, 2007.

18. Sanjiv K, Su TL, Suman S, Kakadiya R, Lai TC, Wang HY, Hsiao M and Lee TC: The novel DNA alkylating agent BO-1090 suppresses the growth of human oral cavity cancer in xenografted and orthotopic mouse models. Int J Cancer 130: 1440-1450, 2012.

19. Lin C, Yang JS, Tsai SC, Lin CF and Lee MR: In vivo evaluation of the synthesized novel 2-benzyloxybenzaldehyde analog CCY-1a-E2 for the treatment of leukemia in the BALB/c mouse WEHI-3 allograft model. Oncol Lett 5: 777-782, 2013.

20. Chang C, Kuo S, Lin Y, Wang J and Huang L: Benzyloxybenzaldehyde analogues as novel adenylyl cyclase activators. Bioorg Med Chem Lett 11: 1971-1974, 2001.

21. Wang JP, Chang LC, Hsu MF, Huang LJ and Kuo SC: 2-Benzyloxybenzaldehyde inhibits formyl-methionyl-leucyl-phenylalanine stimulation of phospholipase D activation in rat neutrophils. Biochim Biophys Acta 1573: 26-32, 2002.

22. Liao CL, Lai KC, Huang AC, Yang JS, Lin JJ, Wu SH, Gibson Wood W, Lin JG and Chung JG: Gallic acid inhibits migration and invasion in human osteosarcoma U-2 OS cells through suppressing the matrix metalloproteinase-2/-9, protein kinase B (PKB) and PKC signaling pathways. Food Chem Toxicol 50: 1734-1740, 2012.

23. Kao TC, Shyu MH and Yen GC: Neuroprotective effects of glycyrrhizic acid and 18beta-glycyrrhetinic acid in PC12 cells via modulation of the PI3K/Akt pathway. J Agric Food Chem 57: 754-761, 2009.

24. Lu CC, Yang SH, Hsia SM, Wu CH and Yen GC: Inhibitory effects of Phyllanthus emblica L. on hepatic steatosis and liver fibrosis in vitro. J Funct Foods 20: 20-30, 2016.

25. Chang PY, Peng SF, Lee CY, Lu CC, Tsai SC, Shieh TM, Wu TS, Tu MG, Chen MY and Yang JS: Curcumin-loaded nanoparticles induce apoptotic cell death through regulation of the function of MDR1 and reactive oxygen species in cisplatin-resistant CAR human oral cancer cells. Int J Oncol 43: 1141-1150, 2013. 
26. Lin CC, Chuang YJ, Yu CC, Yang JS, Lu CC, Chiang JH, Lin JP, Tang NY, Huang AC and Chung JG: Apigenin induces apoptosis through mitochondrial dysfunction in U-2 OS human osteosarcoma cells and inhibits osteosarcoma xenograft tumor growth in vivo. J Agric Food Chem 60: 11395-11402, 2012.

27. Lee CY, Chien YS, Chiu TH, Huang WW, Lu CC, Chiang JH and Yang JS: Apoptosis triggered by vitexin in U937 human leukemia cells via a mitochondrial signaling pathway. Oncol Rep 28: 1883-1888, 2012.

28. Wang JP, Chang LC, Kuan YH, Tsao LT, Huang LJ and Kuo SC: 2-Benzyloxybenzaldehyde inhibits formyl peptide-stimulated increase in intracellular $\mathrm{Ca}^{2+}$ in neutrophils mainly by blocking $\mathrm{Ca}^{2+}$ entry. Naunyn Schmiedebergs Arch Pharmacol 370 353-360, 2004

29. Pan SL, Guh JH, Huang YW, Chang YL, Chang CY, Huang LJ, Kuo SC and Teng CM: Inhibition of Ras-mediated cell proliferation by benzyloxybenzaldehyde. J Biomed Sci 9: 622-630, 2002.

30. Lavrik IN, Golks A and Krammer PH: Caspases: Pharmacological manipulation of cell death. J Clin Invest 115: 2665-2672, 2005

31. Orrenius S: Reactive oxygen species in mitochondria-mediated cell death. Drug Metab Rev 39: 443-455, 2007.

32. Ho TF and Chang CC: A promising 'TRAIL' of tanshinones for cancer therapy. Biomedicine (Taipei) 5: 23, 2015.
33. Kuo HM, Tsai HC, Lin YL, Yang JS, Huang AC, Yang MD, Hsu SC, Chung MC, Gibson Wood W and Chung JG: Mitochondrial-dependent caspase activation pathway is involved in baicalein-induced apoptosis in human hepatoma J5 cells. Int J Oncol 35: 717-724, 2009.

34. Strauss G, Westhoff MA, Fischer-Posovszky P, Fulda S, Schanbacher M, Eckhoff SM, Stahnke K, Vahsen N, Kroemer G and Debatin KM: 4-Hydroperoxy-cyclophosphamide mediates caspase-independent T-cell apoptosis involving oxidative stress-induced nuclear relocation of mitochondrial apoptogenic factors AIF and EndoG. Cell Death Differ 15: 332-343, 2008.

35. Tsai SC, Huang WW, Huang WC, Lu CC, Chiang JH, Peng SF, Chung JG, Lin YH, Hsu YM, Amagaya S, et al: ERK-modulated intrinsic signaling and $\mathrm{G}(2) / \mathrm{M}$ phase arrest contribute to the induction of apoptotic death by allyl isothiocyanate in MDA-MB-468 human breast adenocarcinoma cells. Int J Oncol 41: 2065-2072, 2012.

36. Huang TT, Lin HC, Chen CC, Lu CC, Wei CF, Wu TS, Liu FG and Lai HC: Resveratrol induces apoptosis of human nasopharyngeal carcinoma cells via activation of multiple apoptotic pathways. J Cell Physiol 226: 720-728, 2011. 